\title{
Patient and caregiver involvement in a multicentre clustered hemodialysis trial
}

\author{
Jordan M. Ward MSc, Leah Getchell MA, Amit X. Garg MD PhD; for the MyTEMP Investigators
}

— Cite as: CMAJ 2018;190(Suppl 1):S32-S33. doi: 10.1503/cmaj.180403

$\mathbf{N}$ early 23000 Canadians (about 3 million people worldwide) are currently living with kidney failure and receive maintenance hemodialysis to sustain life. ${ }^{1}$ Patients with kidney failure often require three- to four-hour hemodialysis treatments thrice weekly to clear toxins adequately from their blood and to remove excess fluid. When fluid is removed from the body during hemodialysis (about 1-3 L per session), systolic blood pressure often drops by $20-30 \mathrm{~mm} \mathrm{Hg} .{ }^{2}$ In a six-month period, about $75 \%$ of patients receiving hemodialysis will experience at least one episode of intradialytic hypotension. ${ }^{3}$ These drops in blood pressure are unpleasant for patients (they can lead to muscle cramping, fatigue and nausea) and can cause ischemic injury to the heart and brain. ${ }^{4-6}$ Small studies suggest temperature-reduced dialysis lowers the risk of intradialytic hypotension by up to $70 \%$ compared with standard-temperature dialysis $\left(36.5^{\circ} \mathrm{C}\right) .{ }^{6}$ This may reduce the risk of cardiovascular events and death..$^{6-8}$

It is important to understand the effects of interventions that have the potential to reduce the frequency of drops in blood pressure during hemodialysis, as these frequent hypotensive events are associated with morbidity and mortality. ${ }^{5}$ The needs of patients also require attention, as it has been previously reported that $80 \%$ of clinical hemodialysis research does not address the top research priorities of patients. ${ }^{9}$ Patients receiving hemodialysis have expressed a strong desire to have healthier hearts, as suggested by the following statement: "What are the best ways to promote heart health, including the management of my blood pressure?"10 To understand this better, we developed the Major Outcomes with Personalized Dialysate Temperature (MyTEMP) cluster randomized trial (ClinicalTrials.gov NCT02628366).

This pragmatic trial aims to determine whether patients cared for in hemodialysis centres and randomly assigned to personalized reduced-temperature hemodialysis have better outcomes when compared with patients cared for in hemodialysis centres using a standard dialysate temperature of $36.5^{\circ} \mathrm{C}$ (nonpersonalized). High-priority outcomes for patients, that could be affected by the MyTEMP intervention, were chosen based on evidence. In the intervention arm, a nurse measures the patient's body temperature before the start of each hemodialysis session and sets the dialysis temperature at $0.5^{\circ} \mathrm{C}$ to $0.9^{\circ} \mathrm{C}$ below the patient's predialysis body temperature. The randomly assigned

\section{KEY POINTS}

- A personalized lower dialysate temperature may reduce patients' symptoms related to dialysis and improve cardiovascular outcomes.

- The Major Outcomes with Personalized Dialysate Temperature (MyTEMP) clinical trial aims to determine whether patients cared for in hemodialysis centres and randomly assigned to personalized reduced-temperature hemodialysis have better outcomes than patients cared for in hemodialysis centres using a standard dialysate temperature.

- Discussions with a patient-caregiver partner, as well as insight from the patient and family advisory councils, have led to additional outcomes for analysis and the development of a substudy that focuses on patient symptomology.

- Given that most patients receiving hemodialysis have poor quality of life and life expectancy, their time commitment to patient partnership may be limited; it is important to consider these challenges in scheduling meetings, and in orienting patients who participate on the research team.

treatment (personalized or standard temperature) is delivered by dialysis nurses as it would in routine care. Eighty-four hemodialysis centres in Ontario, which currently care for about 7500 patients, are participating in the MyTEMP trial.

The trial met all Tri-Council Policy Statement (TCPS-2) criteria to waive traditional patient-consent methods where patients typically opt in to a trial. Problems did arise in this process, as local ethic boards had various perspectives on the intervention's minimal risk (a TCPS- 2 criterion for waived patient consent). This was resolved through the presentation of results from pilot studies and systematic reviews, and the argument that reduced dialysate temperature may have occurred in usual care before MyTEMP's implementation. As a waiver for patient consent was granted, MyTEMP was implemented by garnering approval from a unit's medical director to alter the dialysate temperature for all patients; however, both patients and physicians reserve the right to alter the dialysate temperature from this allocated protocol. This method of altered patient consent was granted for all MYTEMP participating sites through various research ethics boards (both local and centralized) across the province. 
We developed and are continuing to conduct MyTEMP guided by patient advice. During the initial study design period of MyTEMP, we presented the trial concept (summary, design, outcomes and follow-up) to several renal patient and family advisory councils (PFACs) across Ontario for open discussion. Through this open dialogue, we obtained input on the trial outcomes of interest and the acceptability of altered methods of patient consent. All PFACs were supportive of the trial design and provided suggestions for other outcomes related to the intervention (e.g., major falls and fractures). The suggestions made by the PFACs were incorporated into the research study. The PFACs also helped improve the readability of a study letter that is provided to patients by each centre's health care team to inform them about MyTEMP, which includes details about how patients can opt out of the trial intervention if they desire to do so. To date, about $90 \%$ of patients have adhered to the randomly allocated therapy. MYTEMP researchers have also routinely corresponded with a caregiver partner associated with our research team. Using insights from the caregiver partner's experience, as well as those early conversations with the PFACs, the MyTEMP team is developing a patient-reported outcome substudy designed to assess outcomes that are highly relevant to patients, and which may be biologically affected by the dialysate temperature (e.g., time to recover from dialysis, pain and tiredness). Patient partners will help inform substudy data collection, the overall trial data analysis, and the knowledge translation strategy to increase the overall impact of MyTEMP.

Hemodialysis-related studies with patient engagement do have unique challenges. Trials, such as MyTEMP, typically require several years of development and follow-up. Patients receiving hemodialysis have a poor prognosis. In some cases, a patient engaged in the project may become too ill to participate or may die. The study team has since developed strategies to address illness and death for patients who are routinely involved in research studies. Strategies include meeting dates that align with the patient's dialysis treatment schedule, and to involve several patients to ensure at least one is available to meet on designated meeting dates. In addition, our patient-caregiver partner serves as a patient liaison, applying her skills as a facilitator and community coordinator. She orients patients to the project, prepares them for meetings, and helps identify and resolve any barriers patients may have participating in the research process.

The new evidence created with MyTEMP has the potential to provide benefits to patient care, survival and well-being (if the hypothesis holds true). It is also giving the renal community valuable experience in leading practices in patient partnership, to best address the existing challenges to producing meaningful research for physicians and patients.

\section{References}

1. Liyanage $T$, Ninomiya $T$, Jha $V$, et al. Worldwide access to treatment for endstage kidney disease: a systematic review. Lancet 2015;385:1975-82.

2. K/DOQI Workgroup. K/DOQI clinical practice guidelines for cardiovascular disease in dialysis patients. Am J Kidney Dis 2005;45(Suppl 3):S1-153.

3. Barth C, Boer W, Garzoni D, et al. Characteristics of hypotension-prone haemodialysis patients: Is there a critical relative blood volume? Nephrol Dial Transplant 2003;18:1353-60.

4. Veljančic L, Popović J, Radović M, et al. Simultaneous blood temperature control and blood volume control reduces intradialytic symptoms. Int J Artif Organs 2011;34:357-64.

5. Flythe JE, Xue H, Lynch KE, et al. Association of mortality risk with various definitions of intradialytic hypotension. J Am Soc Nephrol 2015;26:724-34.

6. Mustafa RA, Bdair F, Akl EA, et al. Effect of lowering the dialysate temperature in chronic hemodialysis: a systematic review and meta-analysis. Clin J Am Soc Nephrol 2016;11:442-57.

7. Sakkas GK, Krase AA, Giannaki CD, et al. Cold dialysis and its impact on renal patients' health: an evidence-based mini review. World J Nephrol 2017;6:119-22.

8. Roumelioti ME, Unruh ML. Lower dialysate temperature in hemodialysis: Is it a cool idea? Clin J Am Soc Nephrol 2015;10:1318-20.

9. Demian MN, Lam NN, Mac-Way F, et al. Opportunities for engaging patients in kidney research. Can J Kidney Health Dis 2017;4:2054358117703070.

10. Manns B, Hemmelgarn B, Lillie EA, et al. Setting research priorities for patients on or nearing dialysis. Clin J Am Soc Nephrol 2014;9:1813-21.
More information on this project is available at www.ossu.ca/ IMPACTAwards.

Competing interests: The authors declare Dialysis Clinic Inc. provided partnership and financial support for the MyTEMP trial with no commercial interest.

This article was solicited and has been peer reviewed.

Affiliations: London Health Sciences Centre (Ward, Garg); patientcaregiver partner (Getchell), London Health Sciences Centre; Departments of Medicine, and Epidemiology and Biostatistics (Garg), Western University, London, Ont.

Contributors: Leah Getchell and Amit Garg contributed to the conception and design of the work, and Jordan Ward contributed to the design of the work. Jordan Ward drafted the article, which Leah Getchell and Amit Garg revised. All of the authors provided final approval of the version to be published and agreed to be accountable for all aspects of the work.
Funding: MyTEMP received funding from partnering organizations, including OSSU (the Ontario SPOR [Strategy for Patient-Oriented Research] SUPPORT [Support for People and Patient-Oriented Research and Trials] Unit), Lawson Health Research Institute, Ontario Renal Network, Dialysis Clinic Inc., Heart and Stroke Foundation of Canada, and Canadian Institutes of Health Research (CIHR). Amit Garg was supported by the Dr. Adam Linton Chair in Kidney Health Analytics and a Clinician Investigator Award from the CIHR.

Acknowledgements: The authors thank Dr. Chris Mclntyre and Ahmed Al-Jaishi for their instrumental involvement in the MyTEMP clinical trial. They also thank all MyTEMP investigators, and other key individuals, for their roles in the development and continuation of the trial.

Correspondence to: Amit Garg, amit.garg@lhsc.on.ca 